\title{
Application of Information Theory in Nuclear Liquid Gas Phase Transition
}

\author{
Y. G. $\mathrm{Ma}^{1,2, \text { ⿵ }}$ \\ ${ }^{1}$ China Center of Advanced Science and Technology (World Laboratory), P. O. Box 8730, Beijing 100080, CHINA \\ ${ }^{2}$ Shanghai Institute of Nuclear Research, Chinese Academy of Sciences, P.O. Box 800-204, Shanghai 201800, CHINA
}

(Dated: PHYS. REV. LETT. 83 (1999) 3617-3620)

\begin{abstract}
Information entropy and Zipf's law in the field of information theory have been used for studying the disassembly of nuclei in the framework of the isospin dependent lattice gas model and molecular dynamical model. We found that the information entropy in the event space is maximum at the phase transition point and the mass of the cluster show exactly inversely to its rank, i.e. Zipf's law appears. Both novel criteria are useful in searching the nuclear liquid gas phase transition experimentally and theoretically.
\end{abstract}

PACS numbers: 25.70.Pq 05.70.Jk 24.10.Pa 24.60.Ky

Hot nuclei can be formed in energetic heavy ion collisions (HIC) and may be highly excited. They deexcite by different decay modes, such as multifragmentation. Experimentally, this kind of multifragment emission was observed to evolve with beam energy (excitation energy, or nuclear temperature, ...). Multiplicity, $N_{i m f}$, of intermediate mass fragment (IMF) rises with the beam energy, reaches a maximum, and finally falls to lower value. This phenomenon of the rise and fall of $N_{i m f}$ may be related to the liquid gas phase transition in nuclear matter [1]. The onset of multifragmentation probably indicates the coexistence of liquid and gas phases. The mass distribution of IMF distribution can be expressed as power law with parameter $\tau$. The minimum of $\tau, \tau_{\min }$, occurs when the liquid gas phase transition takes place [2]. However, $\tau_{\min }$ can also reveal at supercritical densities along the Kertész line [3] and at some subcritical densities at lower temperature fin. So it is not possible to determine the phase transition only from $N_{i m f}$ and $\tau_{\min }$.

On the other hand, experimentalists measured the nuclear caloric curves, i.e. the relationship between nuclear temperature and the excitation energy. $\mathrm{He}-\mathrm{Li}$ isotopic temperature from Albergo thermometer [5] for projectilelike $\mathrm{Au}$ spectators seems to exhibit a temperature plateau in the excitation energy range of 3 to $10 \mathrm{MeV} / \mathrm{u}$ [6]. This plateau was taken as an indication for a first order nuclear liquid gas phase transition. However, due to the changing mass of $\mathrm{Au}$ spectators with excitation energy and the side-feeding effect to measured He- $\mathrm{Li}$ isotopic temperature, this conclusion is questionable [7]. $\mathrm{Nu}-$ clear caloric curves were also surveyed by several groups [8]. However, unfortunately, the sharp signature of liquid gas phase transition in macroscopic systems may be smoothed and blurred due to the small numbers of nucleons in nuclei, and/or the difficulty to perform a direct comparison between the measured "apparent" temperature and the "real" temperature interferes obtaining the real nuclear caloric curve. These factors hamper the

\footnotetext{
*Present Address: Cyclotron Institute, Texas A \& M University, College Station, Texas 77801
}

reaching of a definite conclusion on liquid gas phase transition in nuclei.

The extraction of critical exponents and the study of critical behavior in finite-size systems were attempted in [9] and were followed by controversial debates [10]. In this context, it is necessary and meaningful to search for some novel signatures to characterize the nuclear liquid gas phase transition in order to guide the experimental analysis and theoretical predictions.

In this Letter, we will introduce information entropy [11], $H$, and Zipf's law [12] into the diagnosis of nuclear liquid gas phase transition. The information entropy was defined by Shannon in information theory. Originally, it measures the "amount of information" which is contained in messages sent along a transmission line. It can be expressed as follows,

$$
H=-\sum_{i} p_{i} \ln \left(p_{i}\right) .
$$

where $p_{i}$ is a normalized probability, and $\sum_{i} p_{i}=1$. Jaynes proposed that a very general technique for discovering the least biased distribution of the $p_{i}$ consists in the maximization of the Shannon $H$ entropy, subject to whatever constraints on $p_{i}$ are appropriate to the particular situation. The maximization of $H$ was thus put forward as a general principle of statistical inference one which could be applied to a wide variety of problems in economics, engineering and many other fields, such as quantum phenomena [11]. In high energy hadron collisions, multiparticle production proceeds on the maximum stochasticity, i.e. they should obey the maximum entropy principle. This kind of stochasticity can be also quantified via the information entropy which has been shown to be a good tool to measure chaoticity in hadron decaying branching process [13]. In different physical conditions, information entropy can be expressed with different stochastic variables. In this work on HIC, we define $p_{i}$ as the event probability of having " $i$ " particles produced, i.e. $\left\{p_{i}\right\}$ is the normalized probability distribution of total multiplicity, the sum is taken over whole $\left\{p_{i}\right\}$ distribution. This emphasis is on the event space rather than the phase space. As shown below this kind 
of information entropy [14] can be taken as a method to determine nuclear liquid gas phase transition.

Zipf's law 12 has been known as a statistical phenomenon concerning the relation between English words and their frequency in literature in the field of linguistics. The law states that, when we list the words in the order of decreasing population, the frequency of a word is inversely proportional to its rank 12]. This relation was found not only in linguistics but also in other fields of sciences. For instance, the law appeared in distributions of populations in cities, distributions of income of corporations, distributions of areas of lakes and cluster-size distribution in percolation process 15. In this Letter, Zipf's law will be tested for the fragment mass distribution and it is evidenced to be a factor to characterize the phase transition.

The tools we will use here are the isospin dependent lattice gas model (LGM) and molecular dynamical model (MD). The lattice gas model was developed to describe the liquid-gas phase transition for atomic system by Lee and Yang [16]. The same model has already been applied to nuclear physics for isospin symmetrical systems in the grandcanonical ensemble 17] with a sampling of the canonical ensemble [3, 4, 18, 19, 20, 21, 22], and also for isospin asymmetrical nuclear matter in the mean field approximation [23]. In addition, a classical molecular dynamical model is used to compare its results with the results of lattice gas model. Here we will make a brief description for the models.

In the lattice gas model, $A(=N+Z)$ nucleons with an occupation number $s$ which is defined $s=1(-1)$ for a proton (neutron) or $s=0$ for a vacancy, are placed on the $L$ sites of lattice. Nucleons in the nearest neighboring sites interact with an energy $\epsilon_{s_{i} s_{j}}$. The hamiltonian is written as $E=\sum_{i=1}^{A} \frac{P_{i}^{2}}{2 m}-\sum_{i<j} \epsilon_{s_{i} s_{j}} s_{i} s_{j}$. The interaction constant $\epsilon_{s_{i} s_{j}}$ is chosen to be isospin dependent and be fixed to reproduce the binding energy of the nuclei 20]:

$$
\begin{aligned}
\epsilon_{n n} & =\epsilon_{p p}=0 . \mathrm{MeV} \\
\epsilon_{p n} & =-5.33 \mathrm{MeV} .
\end{aligned}
$$

A three-dimension cubic lattice with $L$ sites is used. The freeze-out density of disassembling system is assumed to be $\rho_{f}=\frac{A}{L} \rho_{0}$, where $\rho_{0}$ is the normal nuclear density. The disassembly of the system is to be calculated at $\rho_{f}$, beyond which nucleons are too far apart to interact. Nucleons are put into lattice by Monte Carlo Metropolis sampling. Once the nucleons have been placed we also ascribe to each of them a momentum by Monte Carlo samplings of Maxwell-Boltzmann distribution.

Once this is done the LGM immediately gives the cluster distribution using the rule that two nucleons are part of the same cluster if $P_{r}^{2} / 2 \mu-\epsilon_{s_{i} s_{j}} s_{i} s_{j}<0$. This method is similar to the Coniglio-Klein's prescription 24] in condensed matter physics and was shown to be valid in LGM [3, 4, 19, 21]. To calculate clusters using MD we propagate the particles from the initial configuration for a long time under the influence of the chosen force. The form of the force is chosen to be also isospin dependent in order to compare with the results of LGM. The potential for unlike-nucleons is

$$
\begin{aligned}
& v_{\mathrm{np}}(r)\left(r / r_{0}<a\right)=C\left[B\left(r_{0} / r\right)^{p}-\left(r_{0} / r\right)^{q}\right] \exp \left(\frac{1}{\left(r / r_{0}\right)-a}\right), \\
& v_{\mathrm{np}}(r)\left(r / r_{0}>a\right)=0 .
\end{aligned}
$$

where $r_{0}=1.842 \mathrm{fm}$ is the distance between the centers of two adjacent cubes. The parameters of the potentials are $p=2, q=1, a=1.3, B=0.924$, and $C=1966$ $\mathrm{MeV}$. With these parameters the potential is minimum at $r_{0}$ with the value $-5.33 \mathrm{MeV}$, is zero when the nucleons are more than $1.3 r_{0}$ apart and becomes strongly repulsive when $r$ is significantly less than $r_{0}$. The potential for likenucleons is written as

$$
\begin{aligned}
& v_{\mathrm{pp}}(r)\left(r<r_{0}\right)=v_{\mathrm{np}}(r)-v_{\mathrm{np}}\left(r_{0}\right), \\
& v_{\mathrm{pp}}(r)\left(r>r_{0}\right)=0 .
\end{aligned}
$$

The system evolves with the above potential. At asymptotic times the clusters are easily recognized. Observables based on the cluster distribution in the both models can now be compared. In the case of proton-proton interactions, the Coulomb interaction can also be added sep- arately and it can be compared with the case without Coulomb effects.

In this Letter we choose the medium size nuclei ${ }^{129} \mathrm{Xe}$ as an example. In most cases, $\rho_{f}$ is chosen to be 0.38 $\rho_{0}$, since the experimental data can be best fitted by $\rho_{f}$ between $0.3 \rho_{0}$ and $0.4 \rho_{0}$ in previous LGM calculations [19, 25], which corresponds to $7^{3}$ cubic lattice. In addition, $0.18 \rho_{0}$, corresponding to $9^{3}$ cubic lattice and $0.60 \rho_{0}$, corresponding to $6^{3}$ cubic lattice of $\rho_{f}$ are also taken to compare and check the results with different $\rho_{f}$ values in the LGM case. 1000 events were simulated for each combination of $T$ and $\rho_{f}$ which ensures enough statistics for results.

In order to check the phase transition behavior in LGM and MD, we will first show the results of some physical observables, namely the effective power-law parameter, $\tau$, 


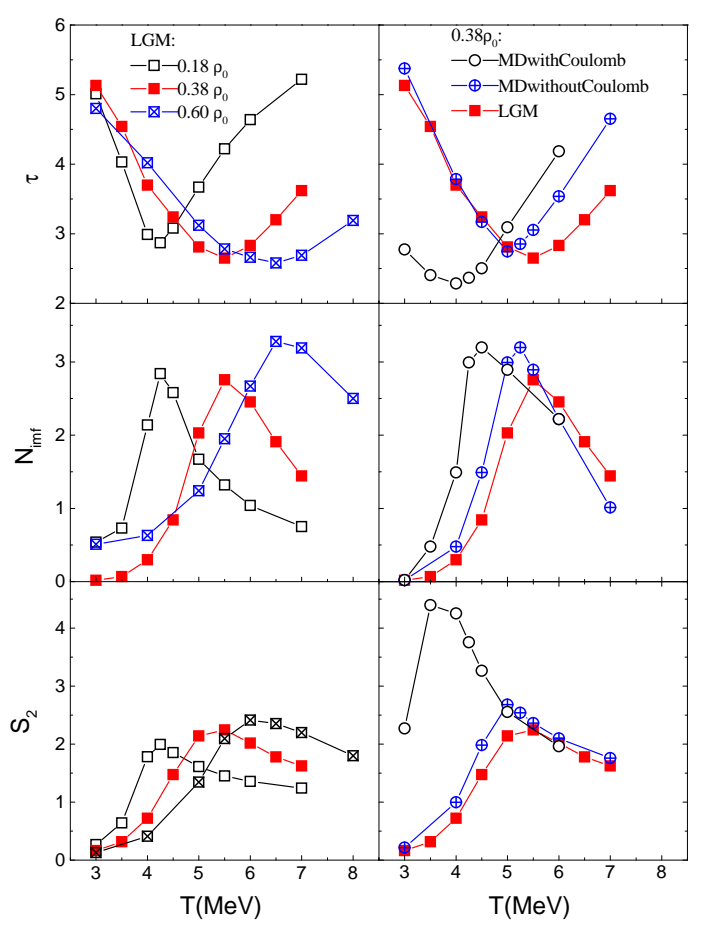

FIG. 1: The effective power-law parameter, $\tau$, the second moment of the cluster distribution, $S_{2}$, and the multiplicity of intermediate mass fragments, $N_{i m f}$ as a function of temperature for the disassembly of ${ }^{129}$ Xe. Left panel is the LGM results with different $\rho_{f}$ and right panel is the comparison of MD to LGM with $0.38 \rho_{0}$. The symbols are illustrated in figure.

the second moment of the cluster distribution, $S_{2}$, and the multiplicity of intermediate mass fragments, $N_{i m f}$, for the disassembly of ${ }^{129} \mathrm{Xe}$ : fig.1. These observables were shown to be good indicators of a liquid gas phase transition, as shown in Ref. [20]. The extreme values of $\tau, N_{i m f}$ and $S_{2}$ occur at the same temperature, indicating of the onset of the phase transition, for each calculation case. For the LGM case, the phase transition temperature increases with the freeze-out density; for the MD case, a slight small transition temperature is obtained when Coulomb force is ignored. It becomes much lower in case of Coulomb interaction due its long range repulsion. Similar phenomenon has been explored in a previous study [20]. However, the aim of this Letter is to take the above transition temperatures as references to search for novel signatures of liquid gas phase transition.

Fig. 2 shows the information entropy for disassembly of Xe. The information entropy exhibits a rise and fall with temperature, which is similar to the behaviors of $N_{i m f}$

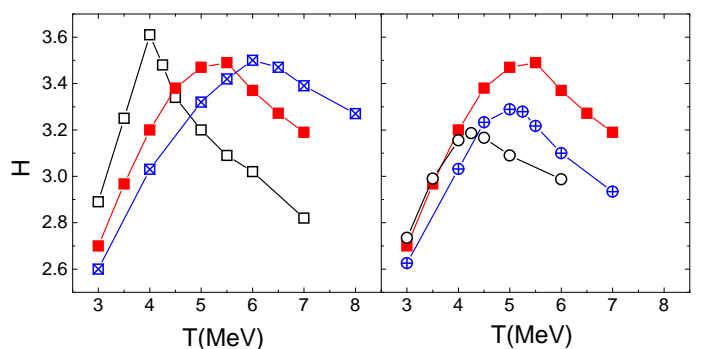

FIG. 2: The same as Fig.1, but for the information entropy $H$.

and $S_{2}$. The temperatures extracted from the peak values of $H$ are consistent with the transition temperatures in Fig.1, indicating that information entropy ought to be a good diagnosis of phase transition. Physically, the maximum of $H$ reflects the largest fluctuation of the multiplicity probability distribution in the phase transition point. In this case it is the most difficult to predict how many clusters will be produced in each event, i.e. the disorder (entropy) of information is the largest. Generally speaking, the larger the dispersal of multiplicity probability distribution, the higher the information entropy and then the disorder of system in the event topology. One should make a careful distinction between this information entropy, on the one hand, and the original thermodynamic entropy, on the other hand 11, 14. The latter generally illustrates the heat disorder in momentum space rather than event space and it always increases with temperature.

Next we will present the results for testing Zipf's law in the fragment distribution. The law states that the relation between the sizes and their ranks is described by $A_{n}=c / n(\mathrm{n}=1,2,3, \ldots)$, where $c$ is a constant and $A_{n}$ is the mass of rank $n$ in a mass list when we arrange the clusters in the order of decreasing size. In the calculations, we averaged the masses for each rank in mass 
lists of the events. Then we examined the relation between the masses $A_{n}$ and their ranks $n$ with the fit of $A_{n} \propto n^{-\lambda}$ in the range of $1 \leq n \leq 10$, where $\lambda$ is the slope parameter. The upper panel of Fig.3 summarizes such parameter $\lambda$ as a function of temperature for LGM and MD cases. Clearly, the value of $\lambda$ decreases with temperature, indicating that the difference of mass between the different fragment ranks is becoming smaller. When $\lambda \sim 1$, the Zipf's law is satisfied: $A_{n} \propto n^{-1}$. The temperatures having Zipf's law for all calculations in Fig.3 are also consistent with the respective transition temperatures extracted from the extreme values of some observables in Fig.1 and 2. Therefore Zipf's law is also a good signal to phase transition. From the statistical point of view, Zipf's law is related to the critical behavior or selforganized criticality [2, 26, which may be a special state with the maximum information or least effort.

In order to further illustrate that Zipf's law exists at the phase transition point most probably, we directly fit the rank-classified cluster distribution with Zipf's law and extract the truth of the hypothesis: $\chi^{2}$ test. The lower panel of Fig.3 demonstrates the $\chi^{2} / n d f$ for the $A_{n}$ - $n$ relations at different $T$ for different calculation cases. As expected, there are minima of $\chi^{2} / n d f$ around the respective transition temperature, which further support Zipf's law of the fragment distribution indicates a phase transition. All calculations give the same conclusions as above.

In addition, we also investigated larger systems, such as $A=274,500$ and 830 , in the LGM case to see if the system behaves as expected in ${ }^{129} \mathrm{Xe}$. The results show that the maximum of information entropy and Zipf's law behavior still remain at the same phase transition temperature as the one extracted from the extreme values of $\tau, N_{i m f}$ and $S_{2}$. It illustrates that both novel criteria are suitable as signals of phase transition in larger $A$ limit.

In conclusion, the information entropy and Zipf's law in the field of information theory are introduced, for the first time, into the study of the liquid gas phase transition of nuclei in the framework of the isospin dependent lattice gas model and molecular dynamical model in $\rho$-T plane. At the point of phase transition, the information entropy of multiplicity distribution is maximum, which indicates that the system at this time has the largest fluctuation/stochasticity/chaoticity in the event space. Meanwhile, the cluster mass show exactly inversely to its rank, i.e. Zipf's law appears. Even thought both criteria are still phenomenological, we believe that they are simple and practicable tools to diagnose the nuclear liquid gas phase transition in experiments and theories. We are waiting for some data analysis on the information entropy and Zipf's law in near future.

It is my pleasure to thank Dr. J. Pan and Dr. S. Das Gupta for providing the LGM and MD codes and Dr. J. Péter for reading the paper. This work was supported by the China's Distinguished Young Scholar

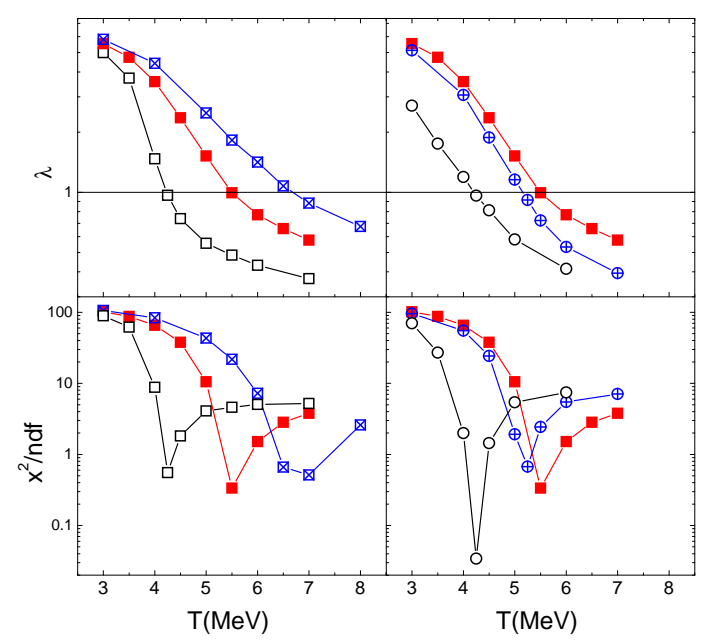

FIG. 3: The slope parameter $\lambda$ of the relation $A_{n}$ to $n$ (upper panel) and the $\chi^{2} / n d f$ with the fit of Zipf's law (lower panel) as a function of temperature. Left panel is the LGM results with different $\rho_{f}$ and right panel is the comparison of MD to LGM with $0.38 \rho_{0}$. The symbols are the same as Fig.1.

Fund under Grant No. 19725521, the NSFC under Grant No. 19705012, the STDF of Shanghai under Grant No. 97QA14038, and the Presidential Foundation of CAS.
[1] C.A. Ogilvie et al., Phys. Rev. Lett. 67, 1214 (1991); M.B. Tsang et al., Phys. Rev. Lett. 71, 1502 (1993); Y.G. Ma and W.Q. Shen, Phys. Rev. C 51, 710 (1995).

[2] M.E. Fisher, Physics (N.Y.) 3, 255 (1967).

[3] X. Campi and H. Krivine, Nucl. Phys. A 620, 46 (1997).

[4] J. Pan and S. Das Gupta, Phys. Rev. C 53, 1319 (1996).

[5] S. Albergo et al., Nuovo Cimento A 89, 1 (1985).
[6] J. Pochodzalla et al., Phys. Rev. Lett. 75, 1040 (1995).

[7] J.B. Natowitz et al., Phys. Rev. C 52, R2322 (1995); M.B. Tsang et al., Phys. Rev. Lett. 78, 3836 (1997); A. Siwek et al., Phys. Rev. C 57, 2507 (1998).

[8] Y.G. Ma et al., Phys. Lett. B 390, 41 (1997); M.J. Huang et al., Phys. Rev. Lett. 78, 1648 (1997); R. Wada et al., Phys. Rev. C 55, 227 (1997); J.A. Hauger et al., Phys. 
Rev. Lett. 77, 235 (1997); V. Serfling et al., Phys. Rev. Lett. 80, 3928 (1998).

[9] M.L. Gilkes et al., Phys. Rev. Lett. 73, 1590(1994); J.B. Elliott et al., Phys. Lett. B 381, 35 (1998); P.F. Mastinu et al., Phys. Rev. Lett. 76, 2646 (1996); M.L. Cherry et al., Phys. Rev. C 52, 2652 (1995).

[10] J.B. Elliot et al., Phys. Rev. C 55, 544 (1997); W. Bauer and A. Botvina, Phys. Rev. C 55, 546 (1997) and references therein; L.G. Moretto et al., Phys. Rev. Lett. 79, 3538 (1997).

[11] K.G. Denbigh and J.S. Denbigh, Entropy in Relation to Uncomplete Knowledge, Cambridge University Press, 1995.

[12] G.K. Zipf, Human Behavior and the Principle of Least Effort, Addisson-Wesley Press, Cambridge, MA, 1949; D. Crystal, The Cambridge Encyclopedia of Language, Cambridge University Press, Cambridge, 1987, p86.

[13] P. Brogueira et al., Phys. Rev. D 53, 5283 (1996); Zhen Cao and R.C. Hwa, Phys. Rev. D 53, 6608 (1996).

[14] The information entropy Eq.(1) is defined only if all events $i$ are a priori equally probable in equilibrium thermodynamics. However, the equal probablity of events is nearly impossible in hadron collisions and nuclear fragmentation since the process is highly dynamical. A theory based on equilibrium thermodynamics would in any case be only an approximation. In this context a phenomenological study is probably more useful than a thermodynamical theory. Of course the entropy defined here is not strict information entropy. Probably, it is better to call it "event entropy" or "multiplicity entropy". However, since the form of information entropy Eq.(1) has been well known, we will still call it information entropy as already made in hadron collision (see 13]) throughout this phenomenological study.

[15] M. Watanabe, Phys. Rev. E 53, 4187 (1996).

[16] T.D. Lee and C.N. Yang, Phys. Rev. 87, 410 (1952).

[17] T.S. Biro et al., Nucl. Phys. A 459, 692 (1986); S.K. Samaddar and J. Richert, Phys. Lett. B 218, 381 (1989); Z. Phys. A 332, 443 (1989); J.M. Carmona et al., Nucl. Phys. A 643, 115 (1998).

[18] W.F.J. Müller, Phys. Rev. C 56, 2873 (1997).

[19] J. Pan and S. Das Gupta, Phys. Lett. B 344, 29 (1995); Phys. Rev. C 51, 1384 (1995); Phys. Rev. Lett. 80, 1182 (1998); S. Das Gupta et al., Nucl. Phys. A 621, 897 (1997).

[20] J. Pan and S. Das Gupta, Phys. Rev. C 57, 1839 (1998).

[21] F. Gullminelli and P. Chomaz, Phys. Rev. Lett. 82, 1402 (1999).

[22] Y.G. Ma et al., Euro. Phys. J. A 4, 217 (1999); J. Phys. G25, 1559 (1999).

[23] S. Ray et al., Phys. Lett. B 392, 7 (1997).

[24] A. Coniglio and E. Klein, J. Phys. A 13, 2775 (1980).

[25] L. Beaulieu et al., Phys. Rev. C 54, R973 (1996).

[26] D. Stauffer, Introduction to Percolation Theory, Taylor and Francis, London, 1985. 